\title{
Adaptive Local Means Filter for Polarimetric SAR Images; Despeckling for Homogeneous and Heterogeneous Clutter Models
}

\author{
Ashraf K. Helmy \\ National Authority for Remote Sensing and Space Sciences, Cairo, Egypt \\ E-mail: ashraf.khaled@narss.sci.eg \\ Ghada S. El-Taweel \\ Suez Canal University, Faculty of Computers and Informatics, Ismailia, Egypt \\ E-mail: ghada@ci.suez.edu.eg
}

\begin{abstract}
Polarimetric radar images suffer from the presence of speckles that degrade the received signal and introduce untruthful indications about the nature of the objects. In this study, we proposed a new framework to filter polarimetric images in which the edges and the channel correlation are preserved. Through a proposed scheme, the image is segmented into groups of regular and irregular pixels. The segmentation process is based on the homogeneity of the texture variation throughout the image. In the homogeneous area, speckle reduction is performed using the adaptive local mean of the neighboring pixels. For non-homogeneous surfaces, the scheme works independently for each set of resolution cells using the general product model containing both intensity and texture information. Quantitative and qualitative assessments confirmed that the proposed filter achieved highly ranked order; it has the ability to preserve fine details, polarimetric information, and to maintain the scattering mechanism of the different objects.
\end{abstract}

Index Terms-Speckle reduction, Synthetic Aperture Radar (SAR), image segmentation, Radar Clutter Model.

\section{INTRODUCTION}

Polarimetric synthetic-aperture radar (POLSAR) is a technology to build an image of an object using a polarized radar signal. Different objects react differently to an incident radar signal. Based on the polarization of an incident wave, the object roughness and its physical characteristics, different objects scatter the incident waves in a manner that indicates the nature of the targets. Using this in satellite remote sensing by transmitting a group of polarized waves and receiving the backscatters, several images can be formed accurately to describe the nature of the objects on the ground.

POLSAR images suffer from speckle noise, one of the major problems with direct effects on the visual interpretation and subsequent image analysis process. Generally, any coherent imaging system (ultra-sound, laser, and SAR) suffers from this phenomenon. It is mainly due to the interference of the backscattering wave from different objects at the receiving antenna. This is a result of the fact that SAR systems record the returned signals coherently to increase the spatial resolution in the flight path direction. This is the origin of the speckle in all SAR systems.

While, the averaging process of a signal contaminated a speckle is the simplest way to remove the speckle, the polarometric SAR data need to be firstly represented as a coherency or covariance matrix [1]. Many authors prove that all useful information can be maintained in a covariance matrix of the original scattering received by the satellite antenna [2-4].

The most direct way to perform an averaging process is the boxcar method that uses a moving window across the whole image, for each pixel, the average value of a sliding window of size $\mathrm{N} \times \mathrm{N}$ pixels replaces the value of the centre pixel. It is considered a blind, low-pass filter [5]. The Lee filter [6] considers only the diagonal elements of the coherency or covariance matrix using the mean square error (MSE). The authors use minimization of MSE through the sliding window. Enhanced LEE [7] uses a local linear minimum mean square error (LLMMSE) estimation within an edge-aligned windows. Only the diagonal elements of the covariance matrix are considered for filtering in IDAN procedures [8]. This approach adopts the surroundings of the pixel under estimation into the local morphology. The algorithm starts by selecting a seed point from the diagonal elements of the covariance matrix. The noise-free pixel is calculated as a mean value of the adopted neighbourhood in the predefined window size.

Recently, Liu et. al. introduced a novel technique based on discrimination between speckles and underlying signal They used a maximum a posteriori probability (MAP) as a measure of separation of speckles [9]. Furthermore, speckle reduction technique is proposed for polarimetric SAR imagery based on filtering only the homogeneous area in a large-scale area while preserving details and fine structure. They assumed that the SAR images follow the Complex Wishart Distribution [10]. Hua Zhong use both nonlocal mean filter to produce the structure similarity 
and the Lee filter to calculate the homogeneity index. Then he uses both measures in a distributive way to conclude a patch similarity measure [11]. Comprehensive overview of despeckling POLSAR image has been introduced in [12] focusing on patch-based methods.

Unfortunately, the previously despeckle methods assume that the POLSAR data follow a "multivariate, zero mean, and complex Gaussian distribution". Consequently, this model fails to describe the scattering of heterogeneous areas [13], where the characteristics of incident wave are altered during wave propagation. The concept of homogeneous/heterogeneous area will be discussed in subsequent sections. The Gaussian model designed for homogeneous surface fails to define the texture information [14], to accommodate a surface heterogeneity, it is necessary to describe the received data using more complex statistical model [14-16].

Generally, in the design of speckle filters, two main aspects should be taken into consideration: we should preserve the correlation between the polarimetric channels, meanwhile the spatial resolution should not be degraded. According to this, we can divide the filtering techniques into two types. The first type maintains the spatial resolution at the expense of polarimetric information [17-18], and it depends on integrating the information from different polarimetric channels to estimate the noise-free images. The other type maintains the coherence between the polarimetric channels of the utmost importance [17-18]; usually, these techniques use the spatial relationship between neighbouring pixels to calculate a noise-free image.

The main objective of this paper is to compromise the two previously mentioned groups in despeckle process, where the correlation between the polarimetric channels and the spatial resolution are maintained. This is achieved by incorporating the homogeneity/heterogeneity properties in describing the underlying image. The proposed method is composed of the following steps. The image is first divided into different groups according to its homogeneity. The different groups are assigned to regular and irregular, and the irregular group is further divided into horizontal regular and vertical regular. The noise-free image is then estimated for each pixel individually according to its neighbours' values and homogeneity. The rest of the paper has been sectioned as follows: Section 2 contains a brief description of POLSAR imagery. Section 3 is devoted to presenting the speckle noise model and an analysis of its characteristics. A proposed model for speckle reduction is introduced in Section 4, the experimental results and evaluation are reported in Section 5. Finally, Section 6 presents the main conclusions derived from the study presented in this paper.

\section{POLARIMETRIC SAR MODEL}

The full POLSAR imaging system emits an electromagnetic wave with two orthogonal polarizations, horizontal and vertical. Then, the receiving antenna collects the backscattering wave for both polarizations simultaneously. Four combinations of orthogonal polarization of transmit and receive are used to build a Sinclair "scattering matrix". The scattering matrix represents the intensity and phase of the backscattered wave, usually the matrix elements are recorded in complex form. The four components of the scattering matrix are $S_{H H}, S_{H V}, S_{V H}$, and $S_{V V}$ where $\mathrm{S}, \mathrm{H}$, and $\mathrm{V}$ refer to the scattering coefficient matrix, horizontal and vertical polarization states, respectively. The scattering matrix can be used for further image analysis.

$$
\mathrm{S}=\left[\begin{array}{ll}
\mathrm{S}_{\mathrm{HH}} & \mathrm{S}_{\mathrm{HV}} \\
\mathrm{S}_{\mathrm{VH}} & \mathrm{S}_{\mathrm{VV}}
\end{array}\right]
$$

Cloude 1996 suggested another method to represent the scattering matrix for better extraction of the physical information [19-20]. His method depends on building a vector system of Sinclair matrix as follows:

$$
\mathrm{y}=\left[\begin{array}{llll}
\mathrm{S}_{\mathrm{HH}} & \mathrm{S}_{\mathrm{VH}} & \mathrm{S}_{\mathrm{HV}} & \mathrm{S}_{\mathrm{VV}}
\end{array}\right]^{\mathrm{T}}
$$

In the remote sensing environment, reciprocal media prevail, which have $S_{H V}=S_{V H}$. This situation simplifies the Sinclair matrix to a three dimensional complex vector that characterizes each resolution cell [3], and it is defined as:

$$
\mathrm{y}=\left[\begin{array}{lll}
\mathrm{S}_{\mathrm{HH}} & \sqrt{2} \mathrm{~S}_{\mathrm{HV}} & \mathrm{S}_{\mathrm{VV}}
\end{array}\right]^{\mathrm{T}}
$$

where ' $\mathrm{T}$ ' denotes the transpose of a vector.

Generally, each element in the complex vector can be considered as an independent complex element of the POLSAR data. Its total power (Span) is calculated as:

$$
\operatorname{Span}(\mathrm{S})=\left|\mathrm{S}_{\mathrm{HH}}\right|^{2}+2\left|\mathrm{~S}_{\mathrm{HV}}\right|^{2}+\left|\mathrm{S}_{\mathrm{VV}}\right|^{2}
$$

To disclose the physical information of the objects for further image analysis and pattern recognition processes, the polarimetric images are usually expressed by $4 \times 4$ mean covariance matrices $(3 \times 3$ in the case of reciprocal assumption) [1]:

$$
\mathrm{C}=\mathrm{yy}^{*}
$$

where $\mathrm{C}$ is the mean covariance matrix, $\mathrm{Y}$ represents the backscattering vector and []$^{*}$ denotes the conjugate transpose of a complex vector.

To conclude, the Sinclair matrix expressed by (1) possesses two types of information. First, the amplitude (intensities) of the three complex vectors expressed in (3) can be represented by the diagonal elements of the covariance matrix in (5). The other type of information resides in the non-diagonal elements of the covariance matrix, which represent the phase differences between the complex vectors. The complex vector derived from the Sinclair matrix and the subsequent covariance matrix characterizes completely the scattering process only in the case of homogeneous area. Conversely, it cannot describe this process for heterogeneous areas [21]. In the following section, we present the POLSAR noise 
characteristics and major trends used for reducing these phenomena.

\section{POLSAR NOISE MODEL}

To construct a SAR Image, many pulses of electromagnetic waves are transmitted and hit a target scene on the earth. The backscatter of each pulse is recorded. When an electromagnetic wave interacts with ground objects, each object scatters the incident wave differently. Within a resolution cell, these scattered signals are added coherently, that is, they add constructively and destructively depending on the relative phases of each scattered pattern. Speckle noise results from this stochastic nature of constructive and destructive interference and is shown as bright and dark dots in the images. On the other hand, the different objects along the earth possess a heterogeneous and dynamic nature i.e. have different physical characteristics, shape and texture; consequently, these behaviours are translated into the POLSAR images. For instance, "anisotropic materials", such as vegetation, scatter the incident wave with one polarization into another polarization (horizontal polarization is converted into vertical polarization); others objects may preserve the polarization of the incident wave. The homogeneity and heterogeneity of the area of earth subjected to radar signal have a great effect in the characterization and modelling of the scattering of the incident wave and have direct effects on image analysis and object identification.

To conclude, the modelling of the POLSAR data from a homogeneity/heterogeneity point of view and the existence of speckle are two different aspects of the same problem. More precisely, a successful speckle filtering process depends mainly on a derived model to describe the polarimetric data. In the following subsections the modelling of homogeneous/heterogeneous area is introduced.

\section{A. Homogeneous clutter model}

Gaussian model is the simplest form to describe the radar clutter, in this the scattering matrix formed by backscattered waves is represented by a mean covariance matrix (5). Mainly the covariance matrix characterizes the scene reflectivity and it is locally modelled by a multivariate zero-mean complex circular Gaussian distribution [17], with a probability density function equal to:

$$
f(Y ; C)=\frac{1}{\pi^{3}|C|} e^{-Y^{* t}} C_{m}^{-1} Y
$$

where $C_{m}$ is the covariance matrix of the scatter matrix $Y$, and the subscript $*$ denotes the complex conjugate of a vector. Performing multilook processing (MLP) on the received scattering signal $(\mathrm{Y})$ improves the signal-tonoise ratio [21]. MLP is an averaging process applied over "L" independent looks of the same image; consequently, MLP produces the sample covariance matrix $C_{Z}$ in each resolution cell.

$$
C_{Z}=\frac{1}{L} \sum_{i=1}^{L} Y_{i} Y_{i}^{*}
$$

$C_{Z}$ shows a Wishart distribution [21]

$$
p\left(C_{Z} / C_{m}\right)=\frac{L^{L p}\left|C_{Z}\right|^{L-p} e^{-L \operatorname{Tr}\left(C_{m}^{-1} C_{Z}\right)}}{\pi^{\frac{p(p-1)}{2}}\left\lceil( L ) \ldots \left\ulcorner(L-p+1)\left|C_{m}\right|^{L}\right.\right.}
$$

In POLSAR filtering analysis, for homogeneous surfaces, the covariance matrix is assumed to be constant from one pixel to the next [21]. To estimate the noise free value of covariance elements, the maximum likelihood (ML) estimate of $\mathrm{C}$ is used, and it is calculated from covariance elements of the all pixels defining the surface [21-24].

\section{B. Heterogeneous clutter model}

The assumption of a homogeneous model for radar scattering leads to lose a significant information regardless of the image texture [1]. To include a texture information in subsequent analysis, the concept of heterogeneous surfaces is required. In this, the element of a covariance matrix $(C)$ has to be calculated for each pixel. the general model to estimate $\mathrm{C}$ on nonhomogeneous areas is introduced as a product model [1], and it is defined as:

$$
\mathrm{C}_{m}=\mu \cdot \mathrm{C}_{\mathrm{h}}
$$

where $\mathrm{C}_{\mathrm{h}}=\mathrm{E}\left(\mathrm{C}_{m}\right)=\mathrm{E}\left(\mathrm{C}_{z}\right)$, the mean covariance of a corresponding homogeneous area, and $\mu$ is the normalized texture factor $(E((\mu)=1))$. This model was extended by [21] using linear minimum mean square estimate (LMMSE) to estimate the covariance matrix $\mathrm{C}_{\mathrm{m}}$ :

$$
\begin{aligned}
\mathrm{C}_{z} & =\mathrm{C}_{\mathrm{F}} \cdot / \mathrm{C}_{\mathrm{R}} \\
\mathrm{C}_{m} & =\mathrm{C}_{\mathrm{h}} \cdot / \mathrm{C}_{\mathrm{R}}
\end{aligned}
$$

where $C_{F}$ is the mean covariance matrix of the Wishart distribution (8), each element of a matrix $C_{R}$ represents the normalized texture value of the corresponding element of C and "./" is a matrix direct product.

$$
C_{R}[i, j]=C[i, j] / C_{F}[i, j]=C[i, j] / E(C[i, j])
$$

The elements of $C_{R}$ are spatially varying with mean $=1$. $\mathrm{C}_{\mathrm{R}}$ and $\mathrm{C}_{\mathrm{F}}$ are independent random variables. The LMMSE estimate of $(8,10)$ estimates $C_{R}$ for each resolution cell of the surface; the estimate of the covariance matrix is calculated by:

$$
\dot{\mathrm{C}}=\dot{\mathrm{C}_{\mathrm{F}}} \cdot / \mathrm{C}_{\mathrm{R}(\mathrm{LMMSE})}
$$

\section{Proposed DENOISING FrAMEWORK}

A proposed framework to reduce speckle in the POLSAR images is shown in Fig. 1. In a proposed method a homogeneous and heterogeneous area are handled differently through speckle removal process; 
accordingly, it is necessary to divide the image into regular and irregular blocks, , each block will be handled differently in speckle removal procedures. The following is a clear operational procedures of the proposed approach.

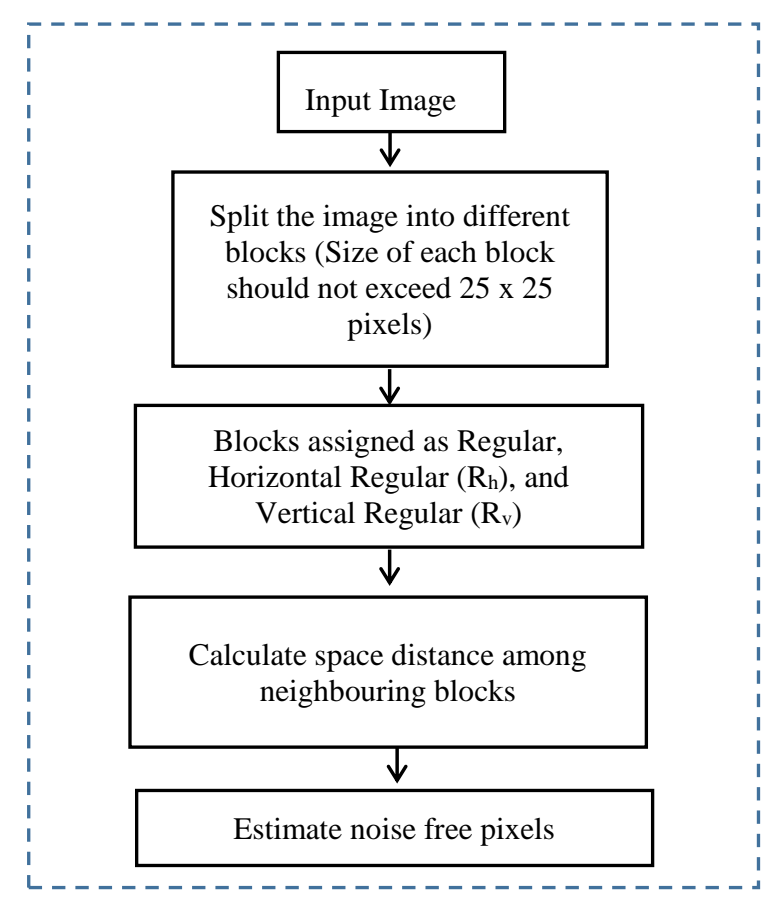

Fig.1. Overall System Diagram for a POLSAR Speckle Reduction

1- The image is divided into several blocks of different sizes, taking into account that each one consists of a group of pixels that have a similar texture "variation". The blocks are assigned to three types (Regular (R), Horizontal Regular $\left(\mathrm{R}_{\mathrm{h}}\right)$, and Vertical Regular $\left(\mathrm{R}_{\mathrm{v}}\right)$ ), depending on the "type" of texture "variation" within the block.

2- We examine the similarity of each block with respect to the surrounding blocks. In other words, we compare the candidate block with centre pixel " $\mathrm{C}_{\mathrm{i}}$ " to all of its neighbour blocks. Structural dissimilarity (DSSIM) was utilized to quantify the space distance between the neighbouring blocks [25].

3- The estimate of the noise-free observation at $\mathrm{C}_{\mathrm{i}}$ is computed as a function of the observations at $\mathrm{C}_{\mathrm{i}}$ added to it as a contribution from neighbouring observations.

In the accompanying sub-sections, we present the different components of the proposed system in more detail.

\section{A. POLSAR image segmentation}

The main goal of this section is to demonstrate the geometry of the POLSAR image through texture flow; this representation provides an idea about the variation of texture throughout the whole image. To achieve our goal, the image is divided into several blocks based on its texture regularity, and entropy (E) is used as a statistical measure of the randomness for each $\operatorname{block}(\Omega)$.

$$
E=-\sum(\mathrm{p} * \log 2(\mathrm{p}))
$$

where each block (p) contains the resolution cell counts. Second, we further divide the block if its entropy shows the highest value (taking into account that the block size should be greater than 11 by 11 pixels).

If the value of entropy is small, then the block is considered to be regularly textured, and we do not split it further. Otherwise, the block is segmented into more subblocks. The quad-tree structure [26] is used for this purpose. For each sub-block, an image gradient and directional change in the intensity are used to indicate whether the sub-block is horizontal or vertical regular. Accordingly, the blocks may be classified into to following types based on block gradient:

Regular (R): texture values in the candidate block have a small value. Regular-horizontal $\left(R_{h}\right)$ : texture has horizontal flow. Regular-vertical $\left(\mathrm{R}_{\mathrm{v}}\right)$ : texture has vertical flow. The limitation of the block size has been chosen experimentally to preserve the structure of a different object within the image. There is no specific rule for this choice. It mainly depends on the nature of the image, and it can be changed from one image to another. The mean segmentation procedure can be summarized as an iterative operation table 1 :

Table 1. Procedures of an Image Segmentation Algorithm

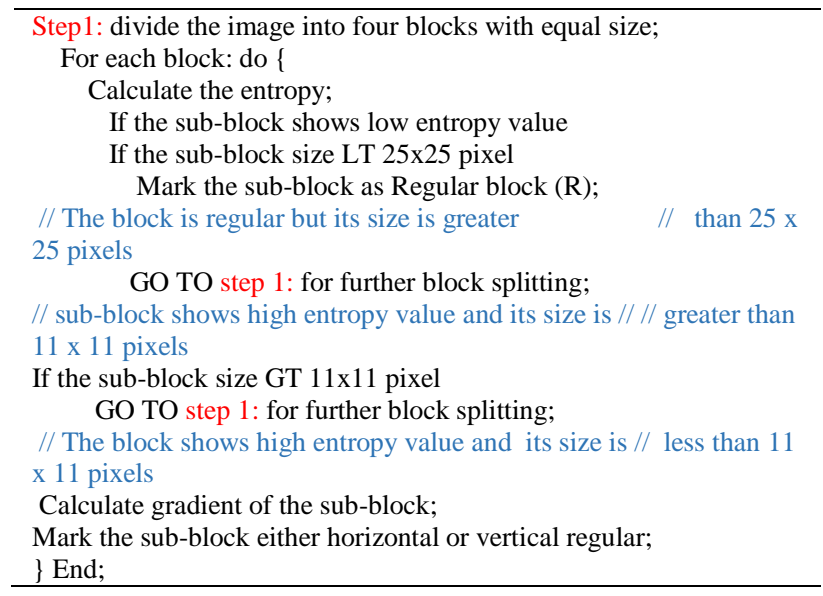

\section{B. Similarity between neighbouring regions}

To assess the extent of similarity of contiguous blocks $(\mathrm{u}, \mathrm{v})$ in the image, structural dissimilarity (DSSIM) was used to measure the distance between adjacent blocks [27] to indicate how close they are to each other, which is determined from the structural similarity index (SSIM)

$$
\operatorname{DSSIM}(u, v)=\frac{1-\operatorname{SSIM}(u, v)}{2}
$$

The SSIM is an index to measure the percentage of similarity between the different blocks of the image. For further clarification, we use the SSIM to show the closeness of each block to adjacent blocks within an image. The measure between two windows $u$ and $v$ in the image is calculated as follows: 


$$
\operatorname{SSIM}(u, v)=\frac{\left(2 \mu_{u} \mu_{v}+c_{1}\right)\left(2 \sigma_{u v}+c_{2}\right)}{\left(\mu_{u}^{2}+\mu_{v}^{2}+c_{1}\right)\left(\sigma_{u}^{2}+\sigma_{v}^{2}+c_{2}\right)}
$$

where:

$\mu_{u}$ and $\mu_{v}$ are the averages of $u$ and $v$; $\sigma_{u}^{2}$ and $\sigma_{v}^{2}$ are the variances of $u$ and $v$; $\sigma_{u v}$ is the covariance of $x$ and $y$;

$c 1$ and $\mathrm{c} 2$ are variables to stabilize the division with a weak denominator. The SSIM values range from -1 to 1 . When its value is equal to one, there is an entire match between the two neighbours in terms of data content. It is usually calculated on window sizes of $8 \times 8$. Then, the window is shifted pixel-by-pixel sequentially. In this study, the authors used the entire sub-block to reduce the complexity of the calculations.

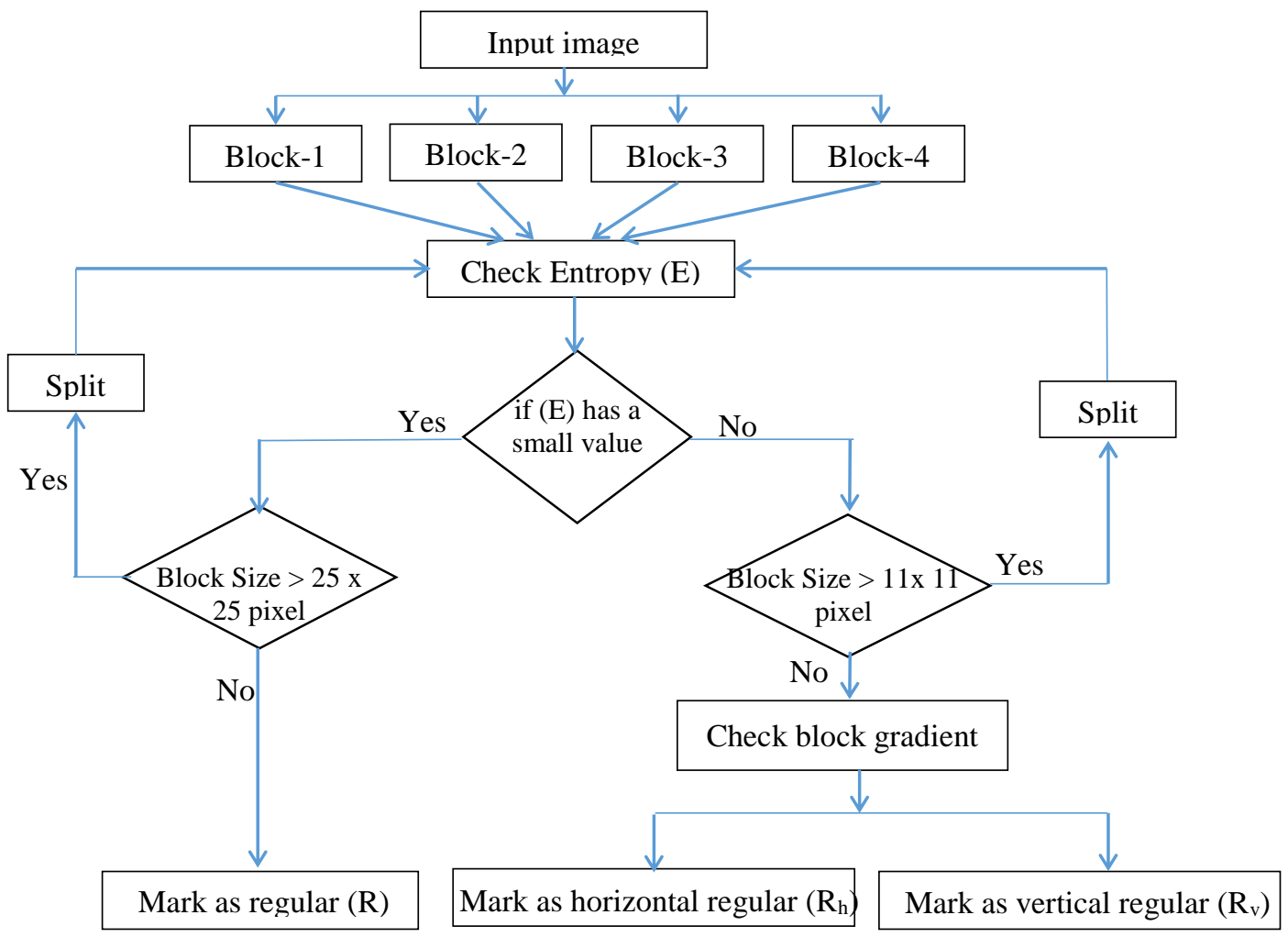

Fig.2. System Diagram for a POLSAR Segmentation Process

\section{Estimation of a noise free image}

Lee, J.S., Pottier characterized the backscatter of the incident radar signal in POLSAR by spherically invariant random vectors model [1]. The data clutter is described by two parameters: the normalized texture and the speckle normalized covariance matrix. As described in the previous sections, for homogeneous areas (case-1), the texture random variable has a constant value in the neighbourhood of a resolution cell, the maximum likelihood (ML) can be used to estimate the free-noise covariance matrix, and is calculated only from all the pixels defining the homogeneous surface [24]. The heterogeneous areas (case-2) can be modelled by some probability density function (PDF). The choice of PDF depends on the degree of irregularity of the surface [21].

In the proposed method, the pixels in the image have been assigned into three categories, namely, regular (R), horizontal regular $(\mathrm{Rh})$, and vertical regular $(\mathrm{Rv})$. Due to the mechanism of the POLSAR interaction with different ground objects, we assume that the irregular area $(\mathrm{Rh}$ and $\mathrm{Rv}$ ) is not affected significantly by scattering from the regular area $(\mathrm{R})$, but the reverse is not true, i.e., the regular area is affected by scattering from the irregular area. In the estimation of the noise free pixel value, we consider the following two cases:

Case-1: for pixels belonging to the regular area (R)

Given the covariance matrix, C, of a POLSAR image, the filtered pixel, $\tilde{C}(x, y)$, is computed as:

$$
\tilde{C}(x, y)=\frac{\sum_{u, v} f(x+u, y+v) w(u, v)}{\sum u, v w(u, v)} \quad u, v \in \in
$$

To determine the weight value, we use the same scheme employed by many researchers by considering the similarity between a centre and its neighbourhood blocks [21, 22]: the more similarity we have, the more influence there is on the candidate pixel. To summarize, we calculate the value of the noise-free pixel for the candidate block using the weighted mean of the pixels in the block plus the influences from neighboured blocks. These influences are based on a similarity measure between adjacent blocks $(\mathrm{S})$ and the spatial distance from 
the centre pixel (D). The impact of " $D$ " obeys the decreasing strength of interactions as the distance increases. where $w(u, v)$ is the weight assigned to the pixel $(u, v)$.

$$
w(u, v)=f(s, D)
$$

With respect to the spatial distance, the value of the weight at distance Di depends not only on D but also on its past values at distance di-1. Commonly, the strength of this influence decreases as the separation of observations increases. To model this situation, one may replace the distance by its sliding mean $\mathrm{z}$ for a window size $\mathrm{m}$.

$$
z_{k}(u, v)=\sum_{i=1}^{m} w_{i}(u, v) d_{k+1-i}
$$

Case-2: for pixels belonging to the horizontal/vertical regular area $\left(R_{h} / R_{v}\right)$

In case 2 , by defining the irregular surface by a normalised convolution matrix and texture random variable $C_{R}$, we estimate the value of the mean covariance, $\mathrm{C}_{Z}$, for each block individually. More specifically, the value of $C_{z}$ for $R_{h}$ has been estimated independently of $R_{v}$ and vice versa. To meet these requirements, we use the linear minimum mean square estimate (LMMSE) of the estimation of $\mathrm{C}_{\mathrm{z}}$ [21] and presented in section 3 .

The LMMSE estimate of $(8,10)$ gives an estimate of $C_{R}$ for each resolution cell of the surface. The estimated value of $C$ is calculated using (12).

$\dot{C}_{\mathrm{F}}$ is calculated as in case 1 , except that the influences come only from neighbour blocks belonging to the same class.

\section{EXPERIMENTAL RESULTS AND ANALYSIS}

Reliability, validity, and effectiveness are the three principal criteria used to evaluate the proposed method for POLSAR denoising. Although visual interpretation is unconvincing for many mathematicians, it is a significant indication of the overall system outputs.

\section{A. Data set}

For evaluation and comparison with other filter techniques, two data sets were used:

a: San Francisco Bay (NASA/JPL), AIRSAR-image, "Lband, four nominal looks, of 10x10 m" spatial resolution, $7000 \times 901$ pixels, available at

"http://earth.eo.esa.int/polsarpro/datasets.html".

b: Radarsat2 of the Canadian Space Agency for the south part of Egypt, C-band, five nominal looks, $7 \times 7 \mathrm{~m}$ spatial resolution. For better image visualization and human interpretation, the images are presented in false colour using Pauli decomposition [22]. The colour combination is $\left|S_{H H}+S_{V V}\right|^{2},\left|S_{H H}-S_{V V}\right|^{2}$ and $2\left|S_{H V}\right|^{2}$, which are assigned to red, green and blue channels, respectively. The two images are shown in Fig. 3.

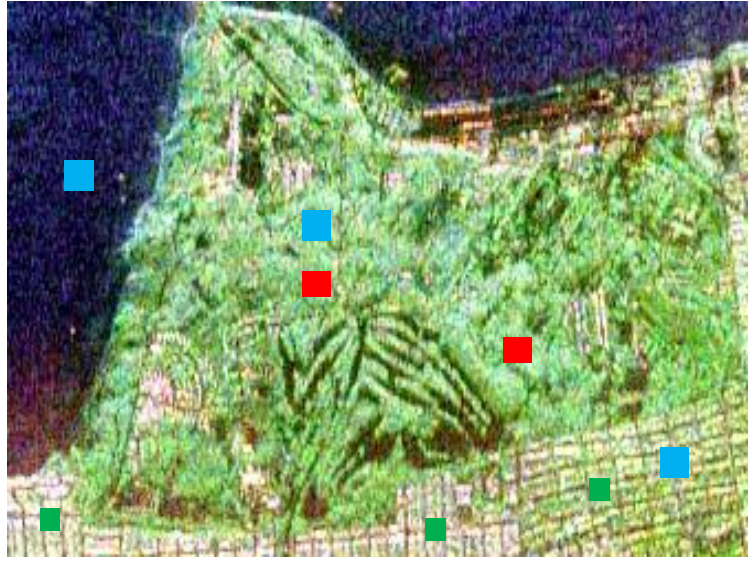

(a)

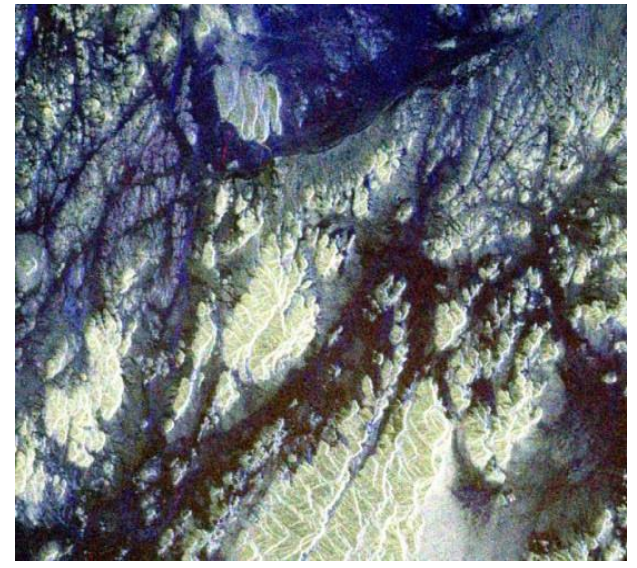

(b)

Fig.3. Pauli decomposition of the original AIRSAR image over San Francisco (a) San Francisco Bay (NASA/JPL) (b) South Part of Egypt, Radarsat2@ EMD

\section{B. Evaluation criteria}

To verify the reliability, which is defined as "the ability of a system to perform its required functions", we visually compare the results with respect to the edges, clarity of different features, and colour diffraction. Generally, any polarimetric filter should not only maintain fine details (edges and tiny targets) but should also avoid interference between polarization channels. Accordingly, some of the performance indices are used to quantify the results in the reliability domain and are discussed below.

1. The Structural Similarity Index (SSIM)

The SSIM measures the similarity between filtered and raw images and its mathematical form is described in section 4-2.

2. Equivalent Number of Looks (ENL).

The value of ENL is used to measure the ability of filters to reduce speckles in SAR images [17]. A higher value of ENL designates weaker speckles. Assuming $\mu$ and $\sigma^{2}$ are the estimated mean and standard deviation, ENL is calculated as 


$$
\operatorname{ENL}(i)=\frac{\mu}{\sigma^{2}}
$$

\section{Ratio of Edge Gradient (REG)}

REG shows the variation of pixel intensities across edges. It is calculated at the boundaries in the image as a ratio between the filtered bands $\left(I_{f}\right)$ and raw image $\left(I_{r}\right)$. This ratio can be calculated either in the $\mathrm{x}$ or $\mathrm{y}$ direction. REG shows the behaviour of intensity changes across the edge and its value is mapped between 0 and 1 . The small value of REG indicates over or under smoothing effects of the filter.

$$
R E G_{v}=\frac{\partial I_{f}}{\partial v_{f}} \overrightarrow{v_{f}} / \frac{\partial I_{r}}{\partial v_{r}} \overrightarrow{v_{r}}
$$

where:

$\frac{\partial I}{\partial v_{f}}$ is the gradient in the $v$ direction for the filtered image. $\frac{\partial I}{\partial v_{r}}$ is the gradient in the $v$ direction for the raw image.

\section{Incoherent Decomposition Parameters}

Cloude and Pottier [12] proposed a method for polarimetric target decompositions. It is directed toward identifying the physical properties of the scattering mechanisms. This method is mostly used for the classification of POLSAR images. It extracts two measures from the scattering covariance matrix, namely, the entropy $(\mathrm{H})$ and the mean alpha angle $(\alpha)$. These parameters are direct representatives of such properties $[1,20]$. The entropy $(\mathrm{H})$ is a way in which the randomness of the scattered signal within a resolution cell is represented; the nature of the scatter can be either "isotropic" $(\mathrm{H}=0)$ or "totally random" $(\mathrm{H}=1)$. The alpha angle differentiates among a medium's scattering mechanisms (volumetric scatter, double bounce, and surface scatter); the value of alpha angle ranges from 0 to 90. Fig. 3 shows the different regions of the h-alpha plane, which provide an interpretation of all random scattering mechanisms for the observed data. We use the twodimensional $\mathrm{H} / \alpha$ (Fig. 4) plane in the analysis of the despeckle filter's performance.

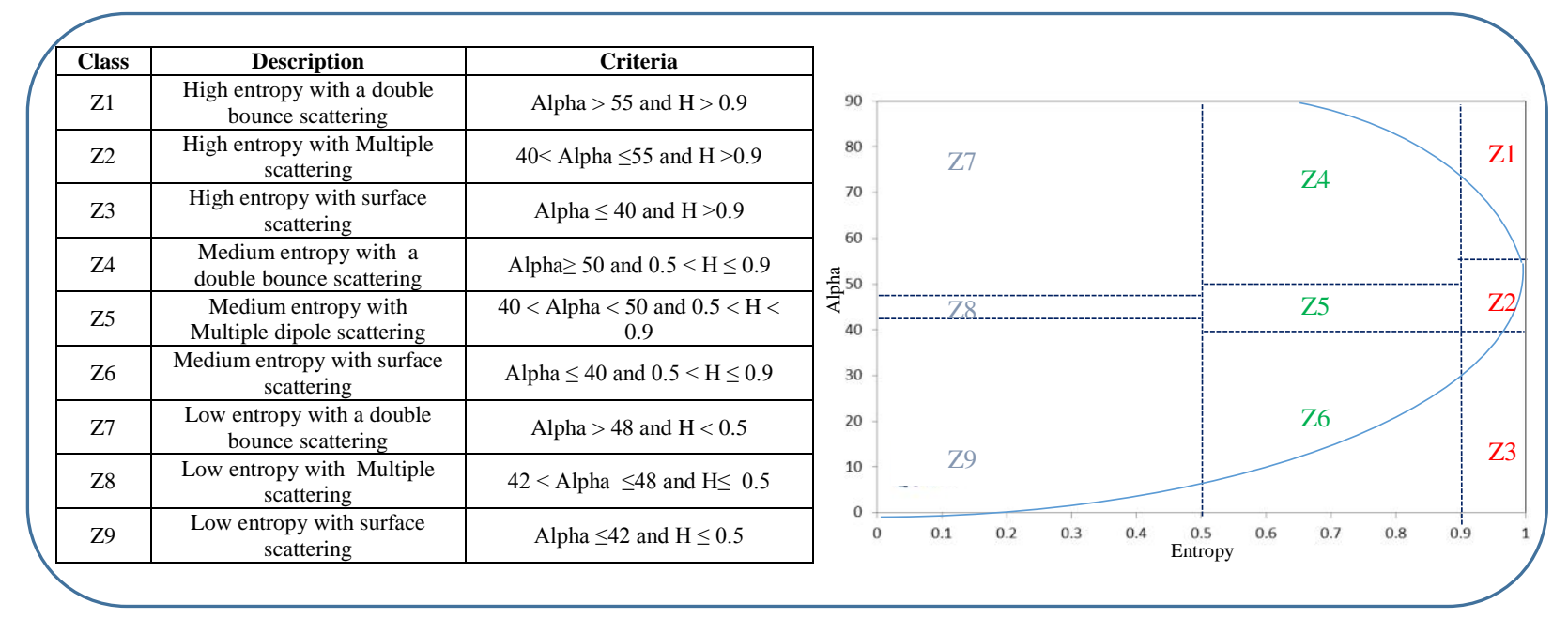

Fig.4. H-alpha Classification Plan @ Cloude and Pottier

\section{Complex Correlation Coefficient $\left(\rho_{c}\right)$ :}

The relation among the off-diagonal elements of the covariance matrix that represents the phase differences between the polarized channels should be preserved through the filtering process. The complex correlation coefficient is a measure of the correlation between polarimetric channels. It is a method to study the preservation of the polarimetric information. The coefficient is measured in the complex form, i.e., the amplitudes and the phase are calculated separately. It is calculated as follows:

$$
\rho_{\mathrm{c}}=\frac{\mathrm{E}\left(\mathrm{S}_{\mathrm{HH}} \mathrm{S}_{\mathrm{VV}}^{*}\right)}{\sqrt{\mathrm{E}\left(\left|\mathrm{S}_{\mathrm{HH}}\right|^{2}\right) \mathrm{E}\left(\left|\mathrm{S}_{\mathrm{VV}}\right|^{2}\right)}}=\left|\rho_{\mathrm{c}}\right| e^{j \theta}
$$

To check the validity of the proposed system with respect to texture information as a random variable for non-homogeneous blocks, we compare the proposed method with the algorithm proposed by Leonardo Torres [24]. Torres' method divides the image into patches with fixed size. He uses the stochastic distance between neighbouring patches for similarity measurement and the non-local mean to estimate the noise-free pixel value; no influence of the texture is introduced in this method. For the effectiveness, we compare our method with three state-of-the-art de-speckle algorithms, namely, the Boxcar, Enhanced Lee and IDAN filters. Brief descriptions of these algorithms are presented in introduction section.

\section{Results and analysis}

The output of the different filters used for assessment is shown in FIG. 5. To validate the usefulness of the various speckle filters, a zoomed-in area of the data is presented in Fig. 6. From the visual effect point of view, the boxcar filter provides excellent noise reduction and 
noticeable edge smoothing. Moreover, it hides some fine details (blue box in Fig. 5-a, 6-b). This has been reported in many studies and is due to the fact that a boxcar filter works as a blind smoother.

Fig. 5-b shows the results of the Enhanced Lee filter. It shows improved filtering effects and preserves edges and fine details, which have strong spatial gradients. This is due to the introduction of the edge-aligned window concept. These results are valid as long as the edges have a high spatial gradient value. When the gradient decreases, some fine details are eliminated, as seen in Fig. 6-c.

The IDAN filter introduces some radiometric losses, as seen in Fig. 5-c, especially for non-homogeneous area. This is due to the strategy used to select the initial seed point. This point is selected as a median of the diagonal elements of the coherency matrix. These points represent irregular patterns with a non-symmetric PDF, so the median and the mean are not the same. As a result, this leads to poor selection of the adaptive neighbourhood. In addition, the IDAN filter fails to preserve some fine details (see for instance Fig. 6-d).

The non-local mean and the proposed method provide similar results with respect to speckle reduction and edge preservation, as shown in Fig. 5-d and e. They have the ability to smooth the image and maintain fine details. A closer look at Fig. 6-e and f (red arrow) shows the nonlocal-mean provides a degraded performance for some structural objects. For further investigations among these methods, we continue with a quantitative analysis.

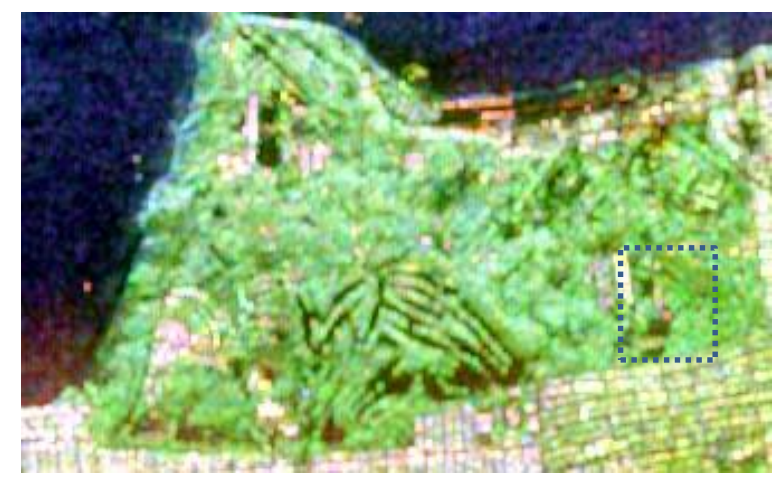

(a)

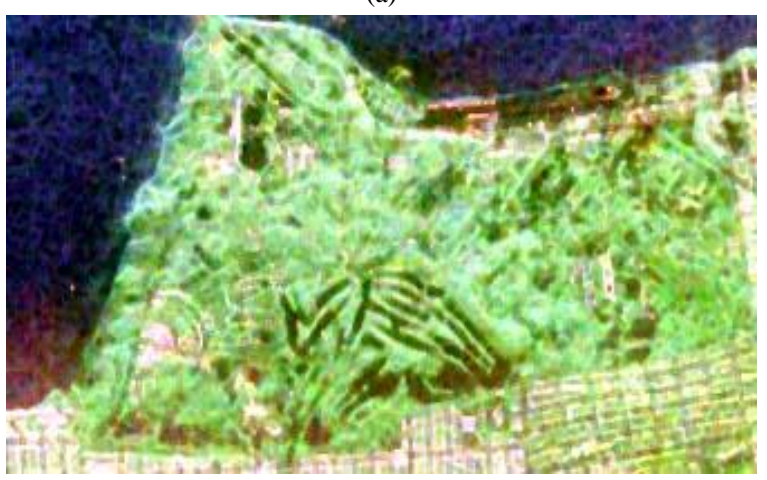

(b)

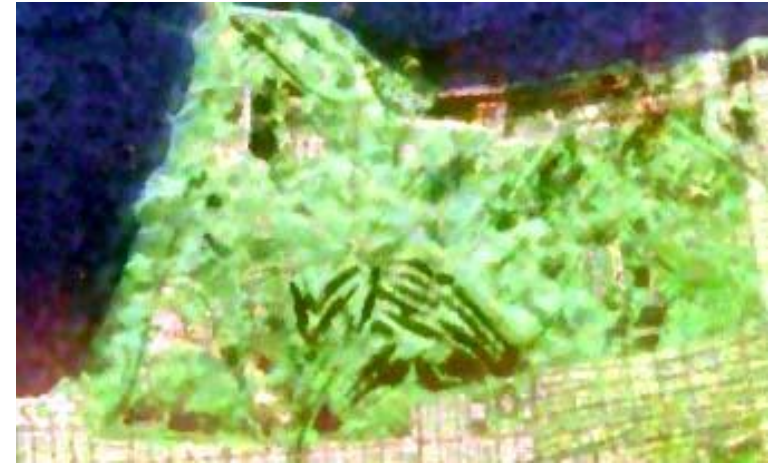

(c)

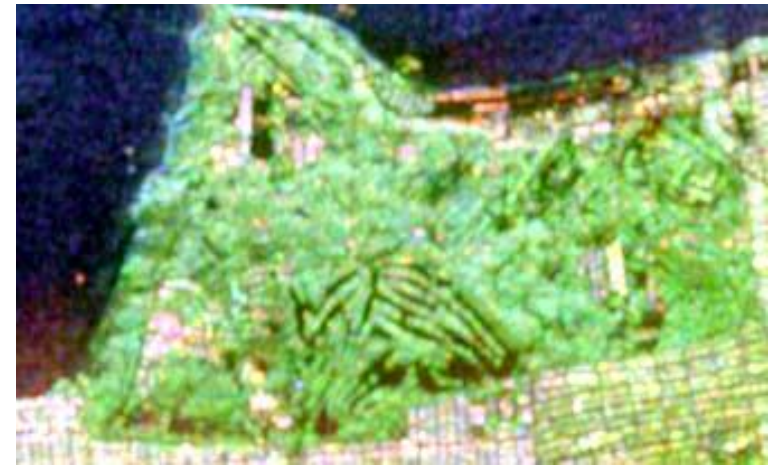

(d)

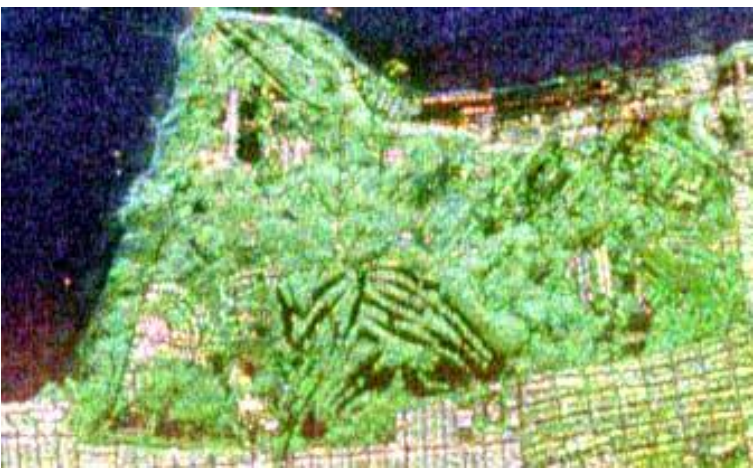

(e)

Fig.5. Comparison of a filter's output on the AIRSAR image over San Francisco.

(a) Boxcar filter; (b) Enhanced LEE filter; (c) IDAN filter; (d) NonLocal mean filter; (e) Proposed filter

During the quantitative analysis, three trends were used in the measurement of the efficiency of the different filters. These trends are summarized as follows:

i. SSIM and ENL are used to show the capability of a filter to reduce the speckle.

ii. For preservation of the polarimetric data through the denoising process, the complex correlation parameter $\rho_{c}$ and the incoherent decomposition parameters are introduced.

iii. Fine details and edge preservation strength are measured using REG. 


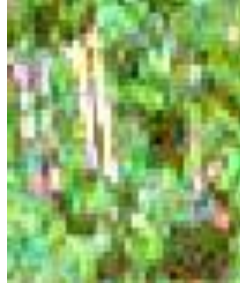

(a)

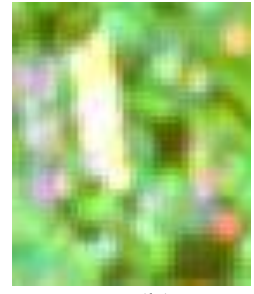

(b)

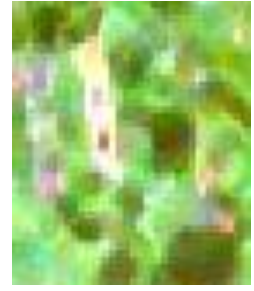

(c)

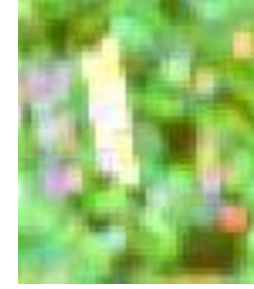

(d)

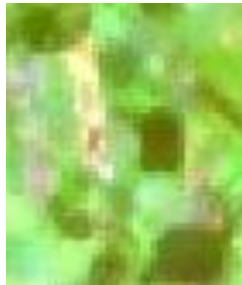

(e)

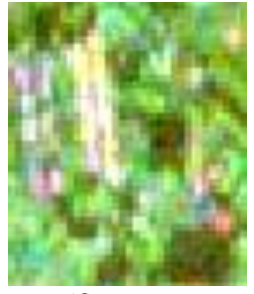

(f)

Fig.6. Zoomed area of the blue box in Fig. 5-a (a) Raw image (b) Boxcar filter; (c) Enhanced LEE filter; (d) IDAN; (e) Non-Local mean filter; (f) Proposed filter

Table 2. Performance measures of the image presented in Fig. 5

\begin{tabular}{|c|c|c|c|c|c|c|c|c|}
\hline & \multirow{2}{*}{ ENL } & \multirow{2}{*}{ SSIM } & \multirow{2}{*}{ REG } & \multicolumn{2}{|c|}{$\begin{array}{c}\text { Incoherent Decomposition } \\
\text { Parameters }\end{array}$} & \multicolumn{3}{|c|}{$\left|\rho_{c}\right|$} \\
\hline & & & & $\mathrm{H}$ & $\alpha$ & ocean & forest & urban \\
\hline Raw image & & & & & & 0.859 & 0.266 & 0.315 \\
\hline Boxcar filter & 19.7 & 0.068 & 0.35 & 4.6 & 0.54 & 0.856 & 0.3 & 0.299 \\
\hline Enhanced Lee & 17.78 & 0.169 & 0.64 & 7.59 & 2.67 & 0.834 & 0.35 & 0.29 \\
\hline Idan & 3.53 & 0.227 & 0.52 & 4.48 & 4.49 & 0.875 & 0.34 & 0.232 \\
\hline Non-local mean & 15.03 & 0.273 & 0.49 & 4.9 & 4.2 & 0.9 & 0.24 & 0.322 \\
\hline Proposed method & 14.9 & 0.2364 & 0.72 & 4.1 & 3.9 & 0.899 & 0.412 & 0.39 \\
\hline
\end{tabular}

Table 2 presents the values of different performance measures, and these results are calculated according to: The ENL and complex correlation coefficient are calculated over the homogeneous areas marked with a red rectangle in Fig. 3. We calculated the REG values over the three edged areas (marked with green rectangles in Fig. 3), and the average values are calculated. The entropy and average angle are calculated in areas representing the sea (where surface scatter is dominant), the vegetation (where volumetric scatter is dominant) and the urban area (where double bounce scatter is dominant). These areas are marked with blue rectangles in Fig. 3.

With respect to the capability of filters to remove speckles from POLSAR images, boxcar yields the best results with the highest value of ENL, and the proposed method ranked fourth. Boxcar and Enhanced LEE are at the end of the list regarding the SSIM index. This coincides with the statement of "the most intense blurring produces the best results with respect to ENL" [24]. The non-local mean and the proposed method have similar (with slight improvements in the non-local mean method) values for SSIM and ENL. This is due to the use of the homogeneous area in the calculations of the non-local mean. For REG, which measures the strength of the filtered edge, the boxcar filter introduces the worst result. This is expected because it works as a blind, low-pass filter. The Enhanced Lee filter comes in second in this index because it uses an edge-aligned window. The proposed method has the best result. Conversely, the IDAN method produces compromised results in all indexes We can conclude that a filter's ability to reduce the speckles comes at the expense of edge and fine detail preservation. For example, boxcar achieves the best capability to remove speckle, but it introduces severe blurring effects. All filters work in an acceptable way to preserve the correlation between polarimetric channels when homogeneity is prevalent. This is clear from the values of the complex correlation coefficients in ocean areas. For the urban and forest areas, where the heterogeneity makes the Gaussian assumption invalid, there is a drop in the complex correlation coefficient values. The proposed method achieves the highest correlation value.

To this extent, the following factors have been considered in our evaluation: polarimetric channels when homogeneity is prevalent. This is clear from the values of the complex correlation coefficients in ocean areas. For the urban and forest areas, where the heterogeneity makes the Gaussian assumption invalid, there is a drop in the complex correlation coefficient values. The proposed method achieves the highest correlation value.

To this extent, the following factors have been considered in our evaluation:

1. Ability of filters to remove speckle, represented by ENL and SSIM.

2. Preservation of edge information and fine details, represented by REG.

3. Interference between polarimetric channels, represented by $\left|\rho_{\mathrm{c}}\right|$.

Complementary with these factors, the entropy $(\mathrm{H})$ and average angles $(\alpha)$ are added in our analysis. We found that it is more persuasive to observe the effect of speckle filtering on the scattering mechanism by examining the H/ $\alpha$ plane. Fig. 7-a shows the original scatters of the three selected areas, namely, ocean, forest, and urban areas. Fig. 7 (b-f) shows the H/ $\alpha$ plane of previously defined objects after being processed by different filters. All of the filters possess a keen ability to converge the scatter of the ocean area and those lying on the zone of low entropy and surface scattering. This is not due to the technique used as much as to the nature of the scattering objects. In urban areas, where multiple scattering is dominant, the different 
filters achieve varying degrees of convergence. The IDAN and proposed method attain the best scatter distribution, whereas the local mean method achieved unacceptable convergence. The same conclusion is valid for the forest area. In the rest of this section, the

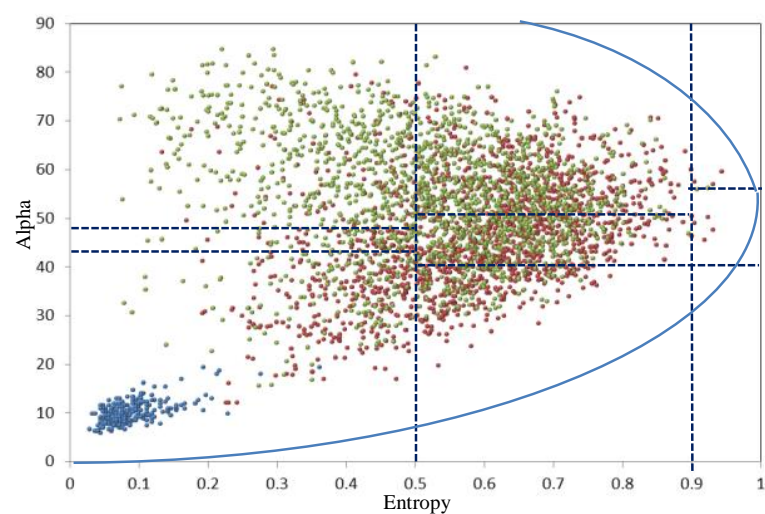

(a) Raw image

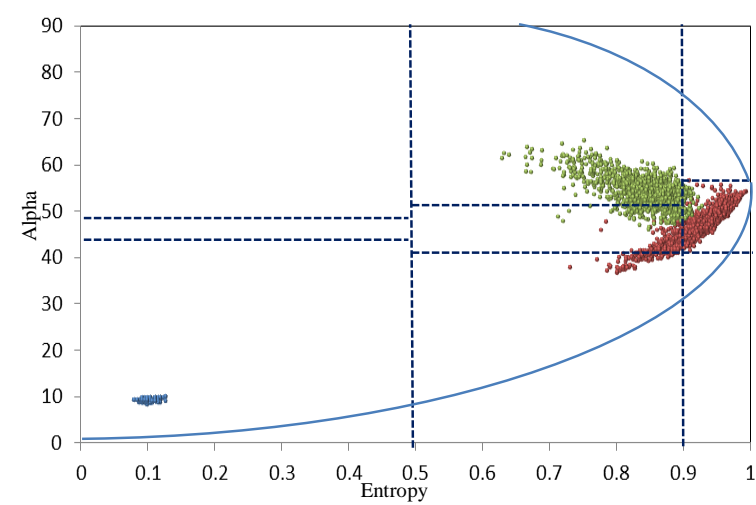

(c) Idan Filter

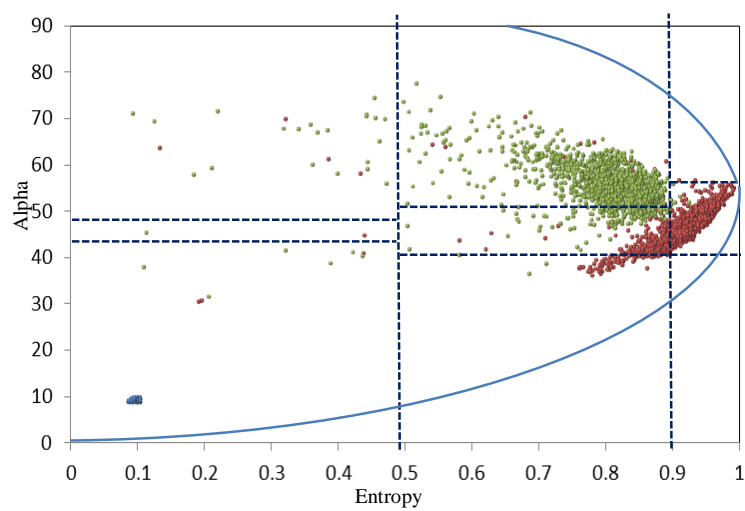

(e) Local mean- Filter preceding analysis is performed on the Radarsat2/C-band (see Fig. 3-b). The output images and results are introduced in Fig. 8 and table 3. The results show an exact match with the results of an earlier analysis.

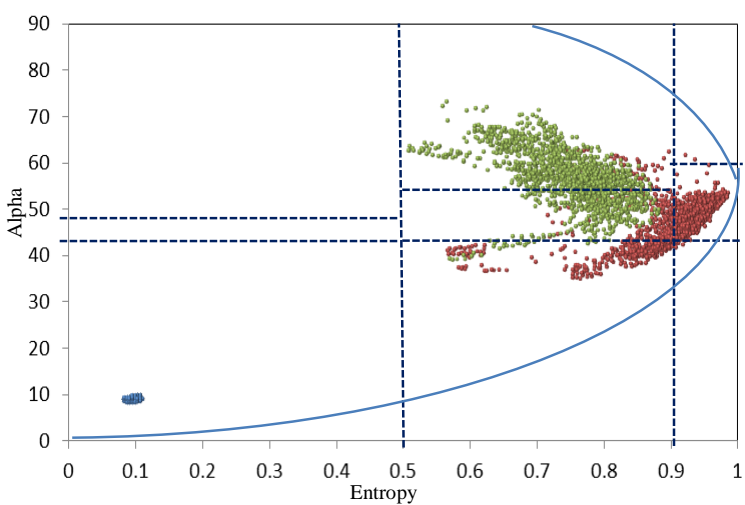

(b) Box Filter

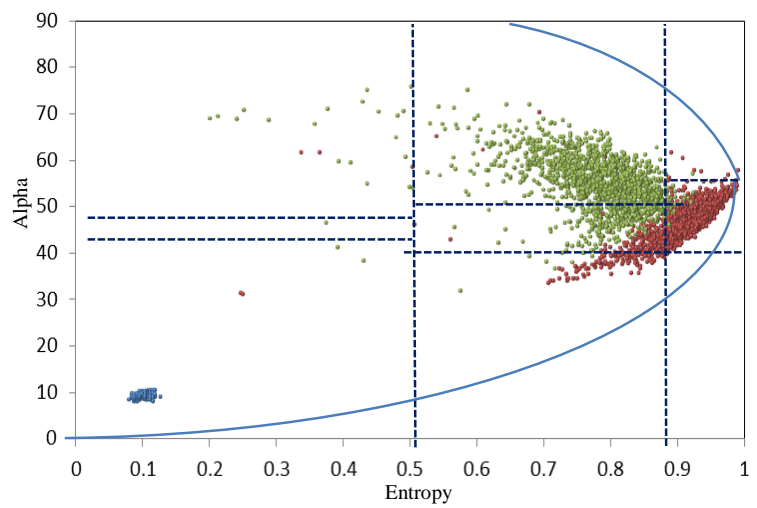

(d) Lee Filter

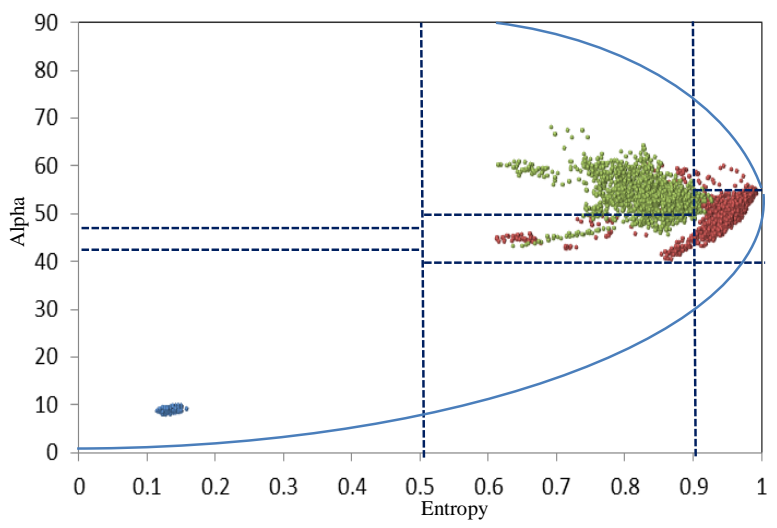

(f) Proposed Filter

Fig.7. Scatter plot in the (H-alpha) plane of samples from the AIRSAR L-band image 
Table 3. Performance measures of the image presented in Fig. 8

\begin{tabular}{|c|c|c|c|c|c|c|}
\hline & \multirow[t]{2}{*}{ ENL } & \multirow[t]{2}{*}{ SSIM } & \multirow[t]{2}{*}{ REG } & \multicolumn{2}{|c|}{$\begin{array}{c}\text { Incoherent } \\
\text { Decompositi } \\
\text { on } \\
\text { Parameters }\end{array}$} & \multirow[t]{2}{*}{$\left|\rho_{\mathrm{c}}\right|$} \\
\hline & & & & $\mathrm{H}$ & $\alpha$ & \\
\hline $\begin{array}{c}\text { Raw } \\
\text { image }\end{array}$ & - & - & - & - & - & 0.289 \\
\hline $\begin{array}{l}\text { Boxcar } \\
\text { filter }\end{array}$ & 25.3 & 0.111 & 0.214 & 5.2 & 0.34 & 0.29 \\
\hline $\begin{array}{c}\text { Enhanced } \\
\text { Lee }\end{array}$ & 19.22 & 0.26 & 0.44 & 6.11 & 3.46 & 0.31 \\
\hline Idan & 14.36 & 0.255 & 0.451 & 5 & 1 & 0.44 \\
\hline $\begin{array}{c}\text { Non-local } \\
\text { mean }\end{array}$ & 14.03 & 0.313 & 0.38 & 5.5 & 4.7 & 0.34 \\
\hline $\begin{array}{c}\text { Proposed } \\
\text { method }\end{array}$ & 17.9 & 0.3341 & 0.66 & 4.99 & 4.09 & 0.492 \\
\hline
\end{tabular}

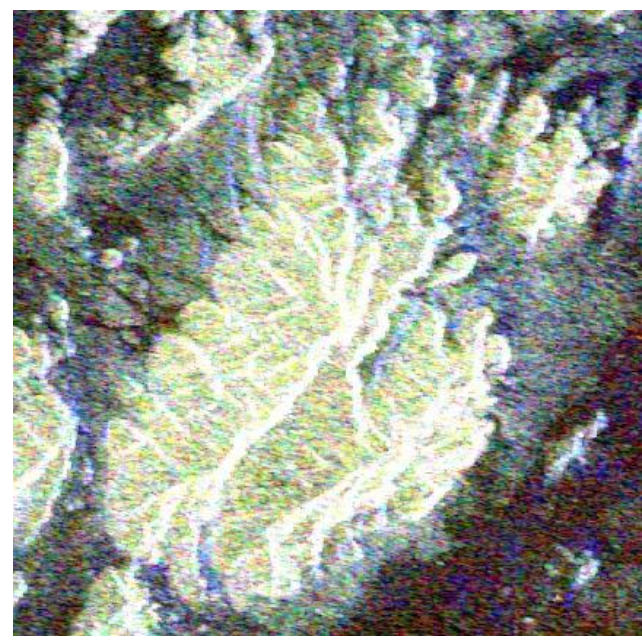

(a)

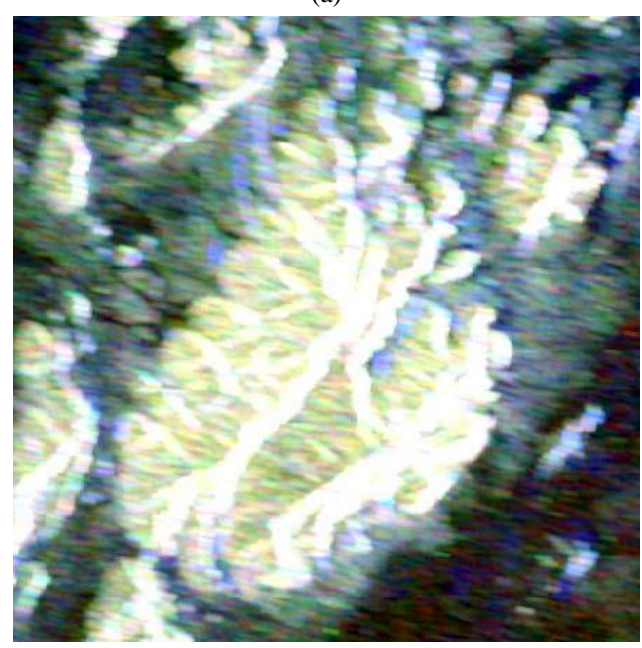

(b)

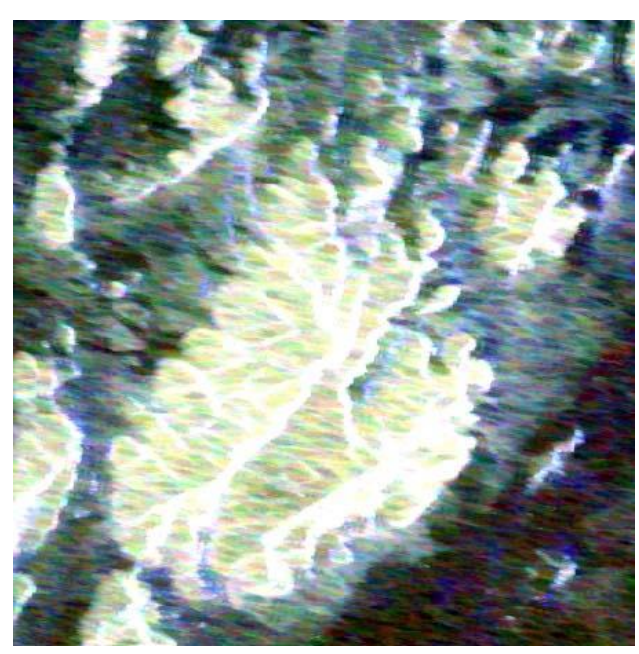

(c)

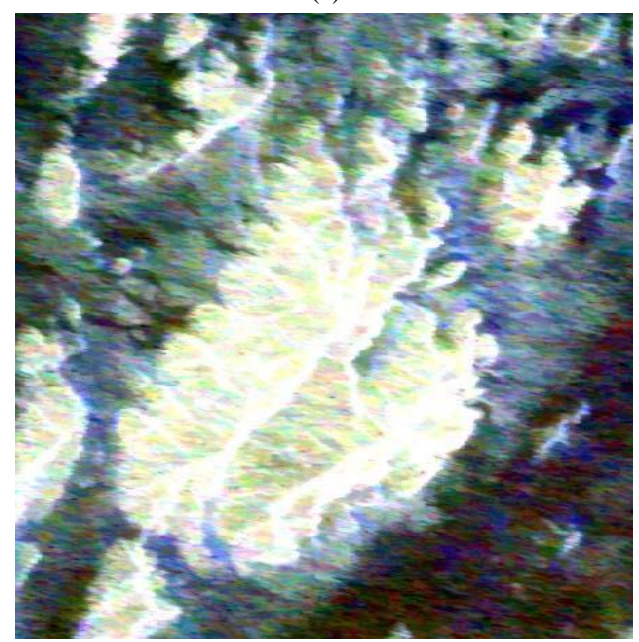

(d)

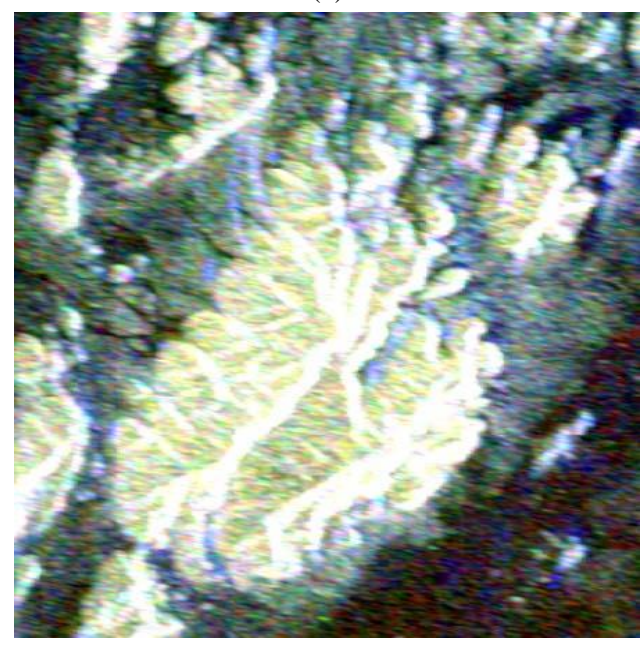

(e) 


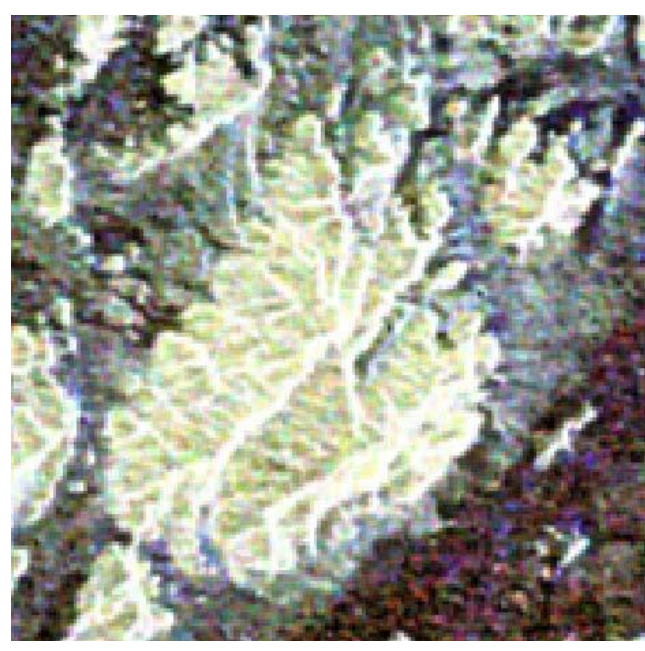

(f)

Fig.8. Comparison of the filter's outputs of the Radarsat 2 images over a part of Egypt (a) Raw image (b) Boxcar filter; (c) Enhanced LEE filter;

(d) IDAN filter; (e) Non-Local mean filter; (f) Proposed filter

\section{CONCLUSIONS}

A new framework in polarimetric Synthetic aperture (POLSAR) speckle filtering has been proposed in this paper. We achieved better harmonize among preservation of fine details, the correlation between polarimetric channels and the image texture. To implement a suggested framework, we proposed an efficient iterative segmentation algorithm in which the image is portioned into regular and irregular regions. Different despeckle models are applied to reduce the speckles according to region regularity.

The proposed framework was compared with The Boxcar, Refined Lee IDAN and non-local mean filters in two data set; AIRSAR-image/L-band and Radarsat2/Cband. BOXCAR filter has great ability to reduce speckle at the expense of other details, Refined LEE shows great ability to reduce speckle, beside it preserves edges and fine details, as longs as it possesses a strong spatial gradient. For IDAN filter, it loses some polarimetric information and fails to maintain small details, especially in urban areas.

The proposed method and nonlocal-mean provide near the performance as long as the investigated area belongs to a homogeneous area, when heterogeneity is introduced to the scene some structural objects are lost in case of non-local mean.

The influence of the filters on scattering mechanism was also investigated using the ( $\mathrm{H}$-alpha). It has been shown that all tested filters introduce denser clusters of observations, especially for smooth areas. On the other hand, it achieves varying degrees of success in rough areas. IDAN and the proposed algorithm have the highest degree of convergence.

\section{ACKNOWLEDGEMENTS}

We express our deepest thanks and appreciation to the Canadian Space Agency (CSA) for providing the
RadarSat 2 images for this study under the agreement of the project ID5128. We also acknowledge the National Authority for Remote Sensing and Space for supporting this work.

\section{REFERENCES}

[1] Lee, J.S., Pottier, E., "Polarimetric radar imaging from basics to applications", CRC Press, Boca Raton, pp. 53-84, 2009

[2] N. R. Goodman, "Statistical analysis based on a certain multivariate complex Gaussian distribution (an introduction)," Ann. Mathemath. Statist., vol. 34, no. 1, pp. 152-177, 1963.

[3] J. Lee, K. W. Hoppel, S. A. Mango, and A. R. Miller, "Intensity and phase statistics of multilook polarimetric and interferometric SAR imagery," IEEE Trans. Geosci. Remote Sens., vol. 32, no. 5, pp. 1017-1028, Sep., 1994.

[4] R. J. A. Tough, D. Blacknell, and S. Quegan, "A statistical description of polarimetric and interferometric synthetic aperture radar data," Proc. R. Soc. Lond. Amer., vol. 449, no. 1937 , pp. 567-589, 1995.

[5] G. Farage, S. Foucher, and G. B. Benie, "Comparison of PoISAR speckle filtering techniques," in Proc. IGARSS, pp. 1760-1763, 2006.

[6] J. S. Lee, M. R. Grunes, and S. A. Mango, "Speckle reduction in multipolarization, multifrequency SAR imagery," IEEE Trans. Geosci. Remote Sens., vol. 29, no. 4, pp. 535-544, Jul. 1991.

[7] J. S. Lee, M. R. Grunes, and G. D. Grandi, "Polarimetric SAR speckle filtering and its implication for classification," IEEE Trans. Geosci. Remote Sens., vol. 37, no. 5, pp. 2363-2373, Sep. 1999.

[8] G. Vasile, E. Trouve, J.-S. Lee, and V. Buzuloiu, "Intensity-driven adaptive-neighborhood technique for polarimetric and interferometric SAR parameters estimation," IEEE Trans. Geosci. Remote Sens., vol. 44, no. 6, pp. 1609-1621, Jun. 2006.

[9] Nonlocal Means Filter for Polarimetric SAR Data Despeckling Based on Discriminative Similarity Measure, Ganchao Liu; Hua Zhong, IEEE Geoscience and Remote Sensing Letters, Vol. 11, Issue: 2, pp: $514-518,2014$.

[10] Jiong Chen; Yilun Chen; Wentao An; Yi Cui; Jian Yang, "Nonlocal Filtering for Polarimetric SAR Data: A Pretest Approach" , IEEE Transactions on Geoscience and Remote Sensing, Vol. 49, Issue: 5, pp. 1744 - 1754, 2011.

[11] Hua Zhong; Jingjing Zhang; Ganchao Liu, "Robust Polarimetric SAR Despeckling Based on Nonlocal Means and Distributed Lee Filter", IEEE Transactions on Geoscience and Remote Sensing, Volume: 52, Issue: 7, pp. 4198 - 4210, 2014.

[12] C.-A.; Denis, L.; Poggi, G.; Tupin, F.; Verdoliva, L., "Exploiting Patch Similarity for SAR Image Processing: The nonlocal paradigm Deledalle", IEEE Signal Processing Magazine, Vol. 31, Issue: 4, pp. 69 - 78, 2014.

[13] R. M. A. Azzam and N. M. Bashara, "Ellipsometry and Polarized Light", Amsterdam, the Netherlands: Elsevier, 1987, pp. 153-416,1987.

[14] L. M. Novak and M. C. Burl, "Optimal speckle reduction polarimetric SAR imagery," IEEE Trans. Aerosp. Electron. Syst., vol. 26, pp. 293-305,1990.

[15] A. Lopés and F. Séry, "Optimal speckle reduction for the product model in multilook polarimetric SAR imagery and the wishart distribution," IEEE Trans. Geosci. Remote Sensing, vol. 35, pp. 632-647, May 1997.

[16] J. S. Lee, M. R. Grunes, and S. A. Mango, "Speckle reduction in multi polarization, multi frequency SAR 
imagery," IEEE Trans. Geosci. Remote Sensing, vol. 29, pp. 535-544, 1991.

[17] Samuel Foucher and Carlos López-Martínez, “Analysis, Evaluation, and Comparison of Polarimetric SAR Speckle Filtering Techniques" IEEE transactions on image processing, VOL. 23, NO. 4, 2014.

[18] J. S. Lee, M. R. Grunes, and G. de Grandi, "Polarimetric SAR speckle filtering and its impact for classification," IEEE Trans. Geosci. Remote Sensing, vol. 37, pp. 23632373, 1999.

[19] J. Schou and H. Skriver, "Restoration of polarimetric SAR images using simulated annealing," IEEE Trans. Geosci. Remote Sensing, vol. 39, pp. 2005-2016, 2001.

[20] Cloude, S.R., Pottier, E.: A review of target decomposition theorems in radar polarimetry. IEEE Transactions on Geoscience and Remote Sensing 34(2), pp. 498-518 1996.

[21] Séry, Franck, and Armand Lopès. "Statistical properties of speckle and full polarimetric filters in SAR." Geoscience and Remote Sensing, IGARSS'97. Remote Sensing-A Scientific Vision for Sustainable Development., 1997 IEEE International. Vol. 2. IEEE, 1997.

[22] Goodman, N.R.," The distribution of the determinant of a complex Wishart distributed matrix. Annals of Mathematical Statistics" vol. 34, pp. 178-180, 1963.

[23] Buades, A., Coll, B., Morel, J.M., "A review of image denoising algorithms, with a new one. Multiscale Modeling \& Simulation”, vol. 4(2), pp. 490-530, 2005.

[24] Leonardo Torres et al, "Speckle Reduction in Polarimetric SAR Imagery with Stochastic Distances and Nonlocal Means" arXiv, pp. 1304.4634 [cs.IT], 2013.

[25] Quegan S. and Rhodes I., "Statistical models for polarimetric data: consequences, testing and validity", IJRS, Vol. 16 no. 7, pp. 1183-1210,1995

[26] Mark de Berg, Marc van Kreveld, Mark Overmars, and Otfried Schwarzkopf, "Computational Geometry" (2nd ed.), Springer-Verlag. ISBN 3-540-65620-0. Chapter 14: Quadtrees: pp. 291-306, 2000.

[27] Z. Wang, A. C. Bovik, H. R. Sheikh and E. P. Simoncelli, "Image quality assessment: From error visibility to structural similarity," IEEE Transactions on Image Processing, vol. 13, no. 4, pp. 600-612, Apr. 2004

\section{Authors' Profiles}

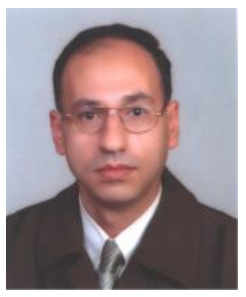

Dr. Ashraf Khaled Helmy is an Associate Research Professor in the National Authority for Remote Sensing and Space Sciences. He is currently the Head of the Space Sciences and strategic studies Division. He has got his Ph.D. in information technology from Cairo University, 2005. His area of interest includes signal and image processing

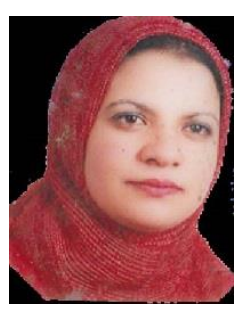

Gh. S. El-Tawel received B.S., M.S., in computer Sciences and Ph.D. degree in Information technology from Cairo University in 1996, 2000, and 2005 respectively. She is currently an associate Professor in Suez Canal University, Ismailia, Egypt. Her research interests include image processing, DNA, and information fusion.
How to cite this paper: Ashraf K. Helmy, Ghada S. ElTaweel,"Adaptive Local Means Filter for Polarimetric SAR Images; Despeckling for Homogeneous and Heterogeneous Clutter Models", International Journal of Information Technology and Computer Science(IJITCS), Vol.8, No.11, pp.33-45, 2016. DOI: 10.5815/ijitcs.2016.11.05 